\title{
Inusual episodio de nevadas de frente cálido en la costa cantábrica y catalana
}

https://doi.org/10.31978/639-19-010-0.487

\author{
Joan Carles Bullón Miró1 (jbullonm@aemet.es) \\ Jaime Fernández García-Moya ${ }^{1}$ (jafernandezg@aemet.es)
}

${ }^{1}$ AEMET / Delegación Territorial en Cataluña

\begin{abstract}
RESUMEN
A finales de febrero de 2018 un anticiclón situado sobre Escandinavia provocó una entrada tardía de aire muy frío de origen polar continental, el cual se fue extendiendo desde Rusia por toda Europa hasta alcanzar la península ibérica, causando más de 40 muertes en el continente. Simultáneamente, entre Islandia y la península del Labrador se generó una profunda borrasca, posteriormente denominada Emma, que se desplazó hacia el sur y después hacia el este y más tarde hacia el norte, pasando sobre Galicia en su movimiento hacia las islas británicas.

La interacción entre esta profunda borrasca y la masa de aire muy frío, que había alcanzado la península ibérica, dio lugar a la formación de un frente cálido y abundantes precipitaciones. Estas fueron inicialmente en forma de nieve en buena parte de la mitad norte peninsular, afectando a cotas muy bajas de la cornisa cantábrica y Cataluña. Son poco habituales las nevadas a cota cero, pero en este caso destaca sobremanera el hecho de que se produjeran por el paso de un frente cálido, lo cual puede ser considerado como hecho excepcional.
\end{abstract}

PALABRAS CLAVE: masa de aire polar continental; borrasca profunda; Emma; nevadas de frente cálido; nevadas a nivel del mar.

\section{INTRODUCCIÓN}

En este artículo se describe la interacción de una masa de aire muy frío de origen polar continental con una profunda borrasca, denominada Emma, que se aproximó a la península ibérica por el oeste. Esta interacción dio lugar a la formación de un frente cálido muy activo sobre la Península, el cual provocó nevadas en su mitad norte a cotas muy bajas. La comparación con las anteriores situaciones meteorológicas que dieron lugar a nevadas a nivel del mar en la costa cantábrica y catalana, demuestra lo extraordinario que fue este episodio.

\section{DESCRIPCIÓN DEL EPISODIO}

\subsection{La extensión por Europa de la masa de aire frío de origen polar continental}

A partir del día 24 de febrero de 2018, la intensificación de un anticiclón situado sobre Escandinavia provocó la extensión de una masa de aire muy frío desde Rusia hacia el oeste, la cual se fue extendiendo progresivamente por Europa central y occidental. 
Esta masa de aire presentaba las siguientes características: 1) fuerte intensidad, con temperaturas a $850 \mathrm{hPa}$ por debajo de $\operatorname{los}-15^{\circ} \mathrm{C}$ en el centro de Europa, generando una corta pero intensa ola de frío en extensas áreas del continente; 2) origen continental, dado que se desplazó desde Rusia hacia el oeste (figura 1); 3) carácter tardío, de manera que el día 28 de febrero fue el día más frío del invierno en Francia (figura 2), país en el cual desde 1947 solo se habían dado tres olas de frío en fechas posteriores al 20 de febrero. Estas propiedades se reflejan en los apelativos que recibió en varios países europeos. Así se le denominó 'La bestia del este' en el Reino Unido, 'Moscú-París' en Francia, 'El oso de Siberia' en los Países Bajos y 'El cañón de nieve' en Suecia. La ola de frío causó más de 40 fallecimientos en Europa, así

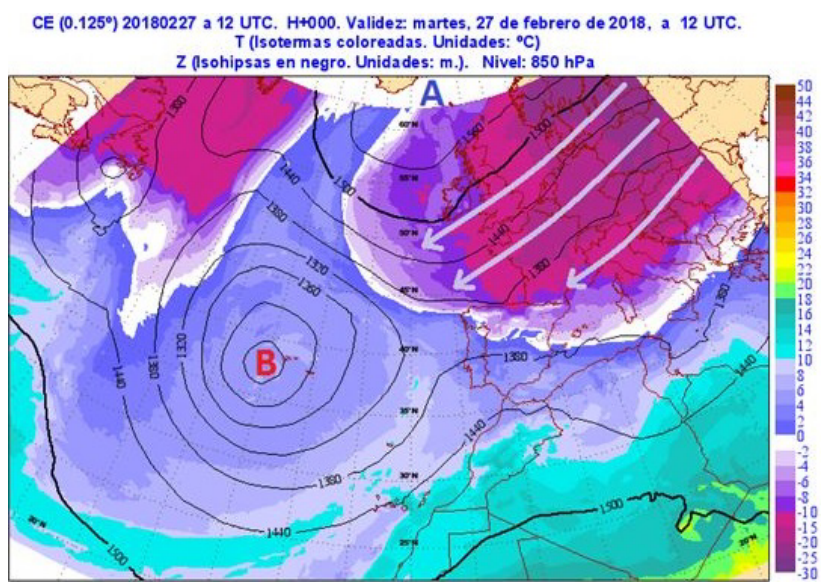

Figura 1. Dirección de desplazamiento de la masa de aire y temperatura a $850 \mathrm{hPa}$ el día 27 de febrero de 2018 a las 12 UTC. como múltiples afecciones.

Aunque en general se trataba de una masa de aire muy fría y seca, en algunas zonas próximas al Mediterráneo, donde era más húmeda, dio lugar a nevadas en lugares donde no son frecuentes, como Venecia, Roma o Nápoles en Italia, o la costa Azul (Niza) y la costa de Córcega en Francia (en Ajaccio no nevaba tanto desde 1985).

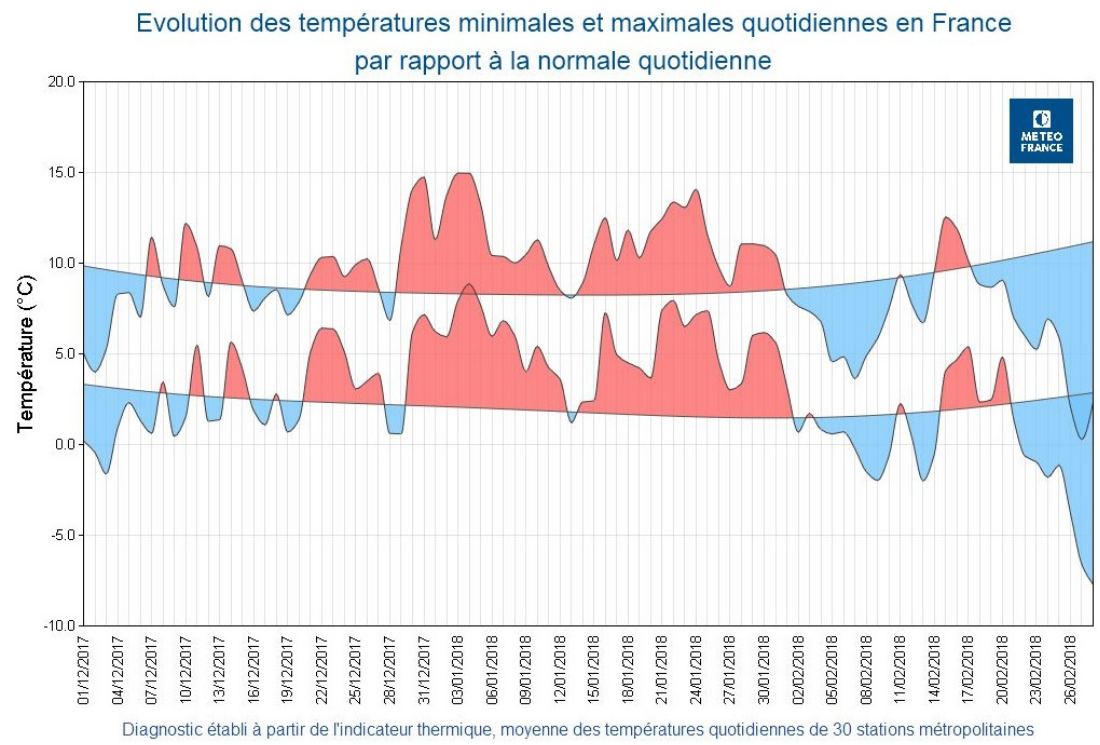

Figura 2.

Media diaria de temperaturas mínimas y máximas en Francia en el invierno 2017-18 (fuente: Meteo France).

La masa de aire polar afectó a la península ibérica más tarde y con menor intensidad y duración que en el resto de Europa, de manera que alcanzó su extremo norte el día 26 de febrero, provocando un descenso térmico, más acusado en Cataluña. El flujo de vientos del norte provocó la habitual formación de un dipolo orográfico por efecto de los Pirineos, lo que dio lugar a vientos del noroeste en el valle del Ebro (cierzo) y del norte en el golfo de León (tramontana). Aunque la masa de aire era poco húmeda, el recorrido marítimo hizo que el aire procedente del golfo de León aumentara su contenido en humedad en niveles bajos. Además, como suele ocurrir cuando se forma este dipolo orográfico, el flujo de vientos dio lugar a convergencias en capas bajas de la atmósfera en el nordeste de Cataluña (figura 3), que causaron nevadas durante la segunda mitad del día en cotas bajas (entre 400-600 m), con acumulaciones de hasta $20 \mathrm{~cm}$. Durante la madrugada del día 27 la cota de nieve continuó bajando y se observaron algunas nevadas en la costa catalana, sin llegar a cuajar. El sondeo de Barcelona del día 27 a las 00 UTC muestra una capa ligeramente inestable y bastante húmeda (figura 4). 

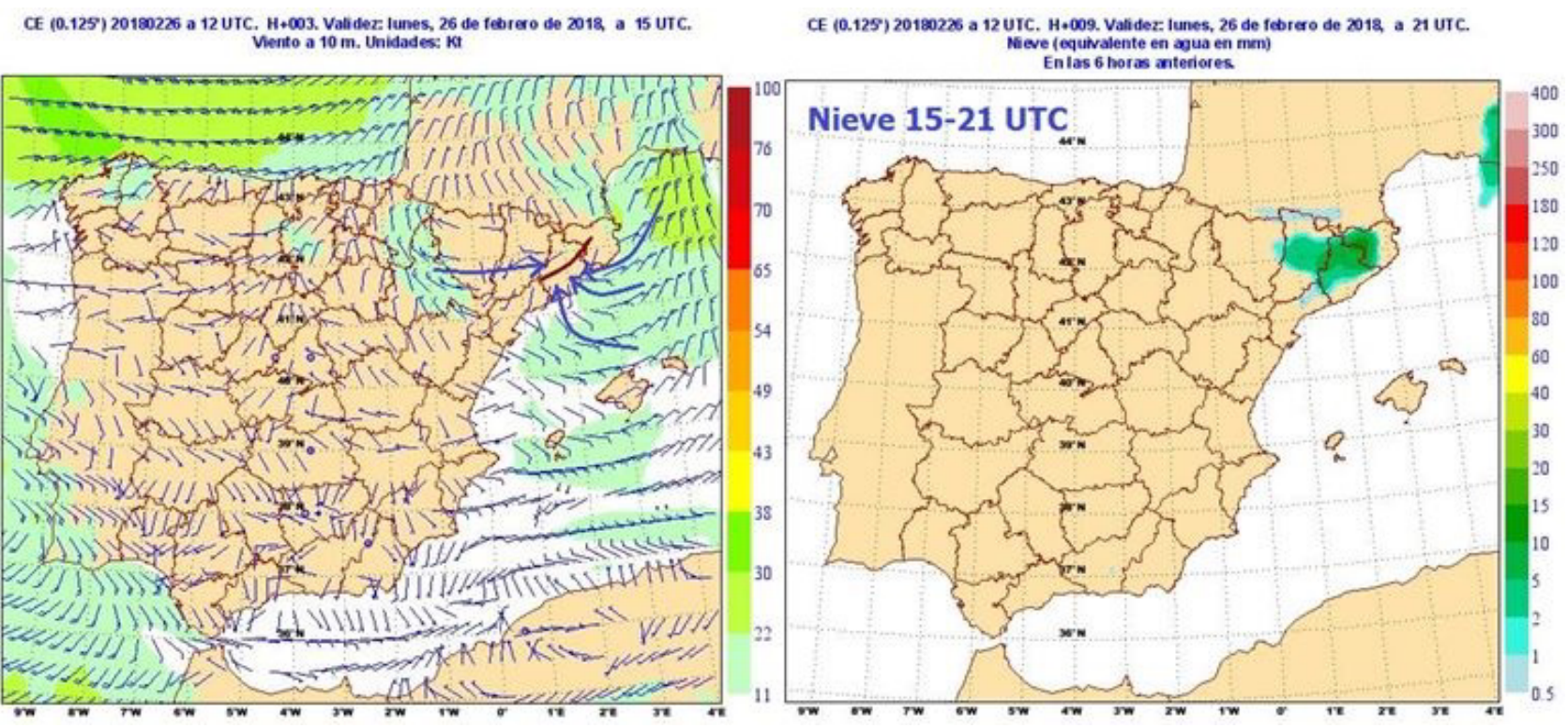

Figura 3. Viento previsto a $10 \mathrm{~m}$ el día 26 de febrero a las 15 UTC (izquierda), donde se indica la zona de convergencias, y precipitación en forma de nieve prevista entre las 15 y las 21 UTC del día 26 (derecha).

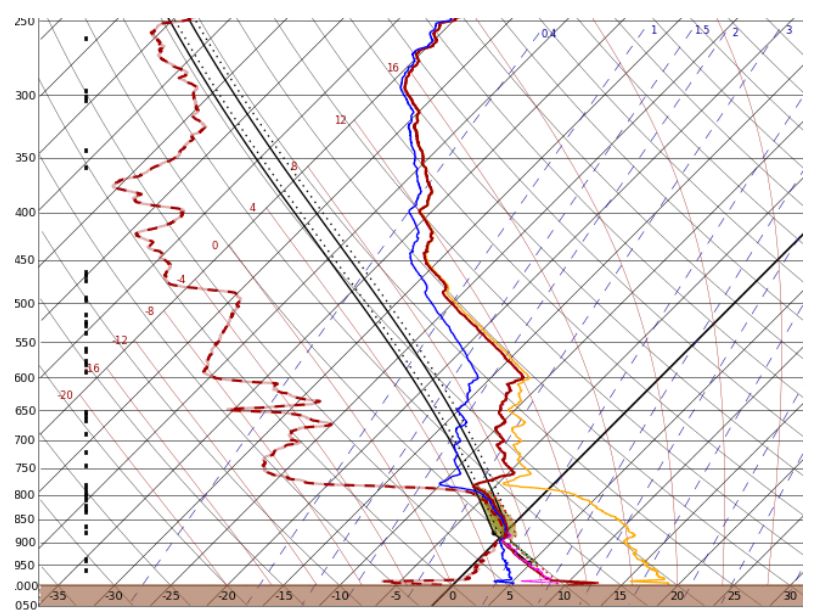

Figura 5.

Descenso de temperaturas en $24 \mathrm{~h}$ a las 14 UTC del día 27 de febrero.
Figura 4.

Sondeo de Barcelona del día 26 a las 12 UTC, donde se aprecia una capa húmeda e inestable entre 900 y $800 \mathrm{hPa}$ aproximadamente.

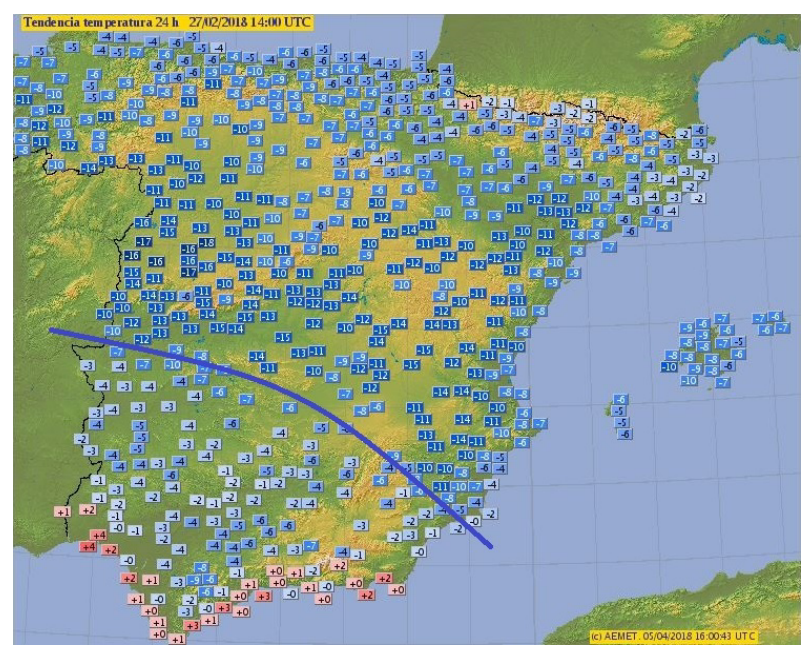

El día 27 la entrada fría alcanzó su máxima extensión sobre la península ibérica, provocando un importante descenso térmico de entre 10 y $15^{\circ} \mathrm{C}$ en amplias zonas del centro peninsular o incluso superior (figura 5). Solo el tercio sur se vio poco afectado por la bajada de temperaturas.

\subsection{La borrasca Emma y su interacción con la masa de aire polar continental}

Una depresión situada entre la península del Labrador y Groenlandia comenzó a profundizarse y desplazarse el día 24 de febrero hacia el sur. Ante la intensidad de los vientos previstos, se la denominó Emma por el servicio meteorológico portugués. 
La borrasca Emma se encontró con el bloqueo del aire polar muy frío que avanzaba por Europa hacia el Atlántico norte, de manera que se vio forzada a desplazarse hacia el sur. Muy activa y con reflejo a todos los niveles de la troposfera, más tarde se desplazó hacia el este en dirección hacia las islas Canarias, para posteriormente moverse hacia el norte, pasando sobre Galicia en su movimiento hacia las islas británicas (figura 6).
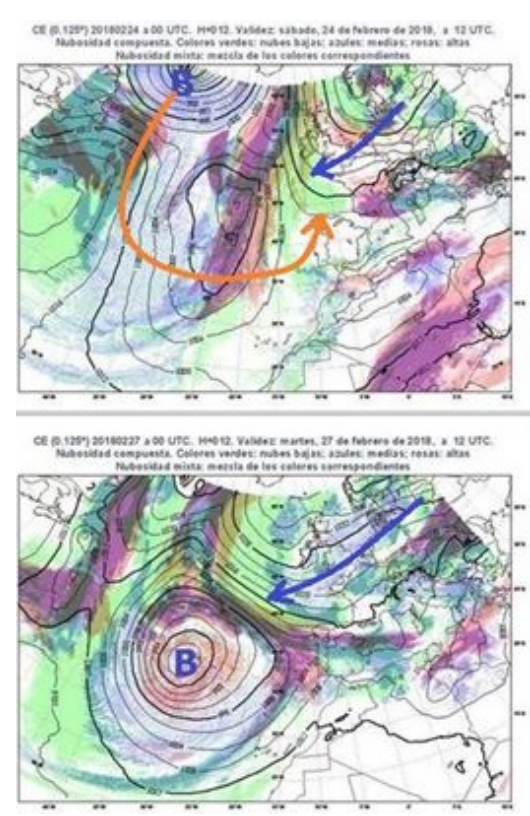
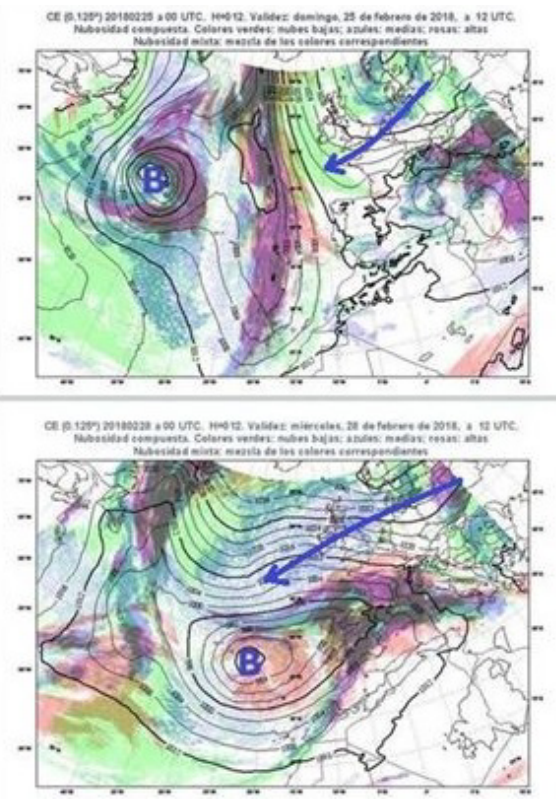
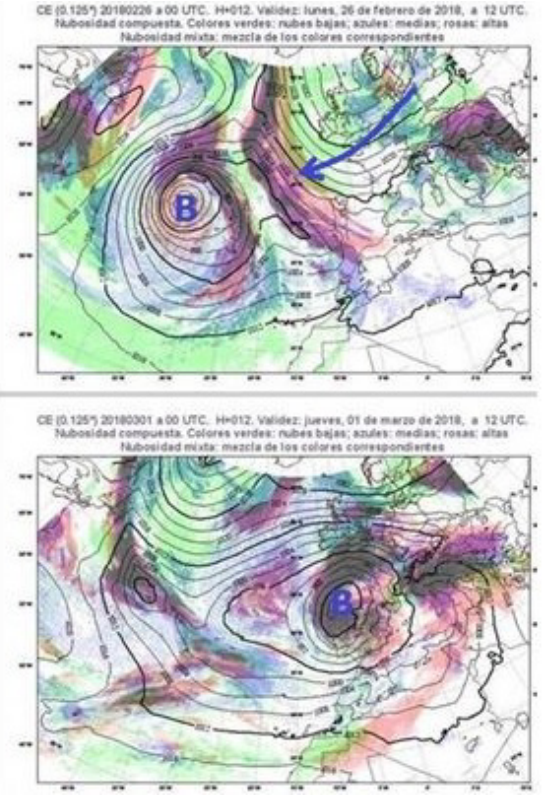

Figura 6. Desplazamiento de la borrasca Emma afectado por su interacción con el bloqueo de la masa de aire polar. Los mapas muestran la presión a nivel del mar y la nubosidad prevista a las 12 UTC entre los días

26 de febrero (arriba a la izquierda) y 1 de marzo (abajo a la derecha). En la figura superior izquierda la flecha roja indica el recorrido que siguió la borrasca.

La profunda depresión Emma dio lugar a vientos muy intensos y a precipitaciones muy abundantes. Como muestra de ello, en la tabla 1 aparecen los registros más elevados de precipitación y rachas de viento en la red de estaciones de AEMET.

\begin{tabular}{|lcc|}
\hline \multicolumn{1}{|c|}{ Estación } & Precipitación acumulada & $\mathrm{mm}$ \\
\hline Puerto El Pico & Provincia & 170,4 \\
Vega de San Mateo & Ávila & 142,8 \\
Madrigal de la Vera & Las Palmas & 126,4 \\
Cazalla de la Sierra & Cáceres & 123,2 \\
Garganta de la Olla & Sevilla & 121,4 \\
Almadén de la Plata & Cáceres & 117,8 \\
San Bartolomé Tirajana, Las Tirajanas & Sevilla \\
La Orotava, Cañadas Teide & Las Palmas & 111,8 \\
Monesterio & Santa Cruz de Tenerife & 110,4 \\
Candeleda & Badajoz & 107,8 \\
\hline
\end{tabular}

Precipitación acumulada máxima durante el día 28

\begin{tabular}{|lccc|}
\hline & Racha $(\mathrm{km} / \mathrm{h})$ & Hora & \\
\hline \multicolumn{1}{|c|}{ Estación } & Provincia & $\mathrm{km} / \mathrm{h}$ \\
\hline Vallehermoso, Alto Igualero & Santa Cruz de Tenerife & $17: 40$ & 166 \\
Izaña & Santa Cruz de Tenerife & $19: 30$ & 147 \\
Agulo & Santa Cruz de Tenerife & $22: 10$ & 127 \\
Valverde & Santa Cruz de Tenerife & $21: 00$ & 125 \\
La Palma Aeropuerto & Santa Cruz de Tenerife & $23: 20$ & 122 \\
Tejeda, Cruz de Tejeda & Las Palmas & $18: 00$ & 121 \\
Agaete- Suerte Alta & Las Palmas & $19: 30$ & 116 \\
Agüimes & Las Palmas & $22: 50$ & 113 \\
Arico & Santa Cruz de Tenerife & $20: 10$ & 111 \\
San Bartolomé Tirajana, Lomo Pedro Alfonso & Las Palmas & $17: 20$ & 110 \\
\hline
\end{tabular}

\section{Rachas máximas de viento durante el día 28, todas ellas en Canarias}

Tabla 1. Valores máximos de precipitación (arriba) y viento (abajo) durante el día 28 en

la red de estaciones meteorológicas de AEMET. 


\subsection{Nevadas de frente cálido en la mitad norte de la península ibérica}

Como se ha comentado, el día 27 de febrero la masa de aire frío alcanzó su máxima extensión sobre la península ibérica. La aproximación de la borrascaEmma frenó su avance, debido a que el aire más cálido y húmedo empujado por ella, procedente del Atlántico, formó un marcado frente cálido, que avanzó en dirección sur-norte (figura 7). El frente dio lugar a nevadas en la mitad norte peninsular en cotas muy bajas.

Los sondeos en Santander muestran el importante descenso térmico que tuvo lugar el día 27 en capas bajas de la atmósfera y el posterior ascenso térmico provocado por el frente cálido. El sondeo del día 28

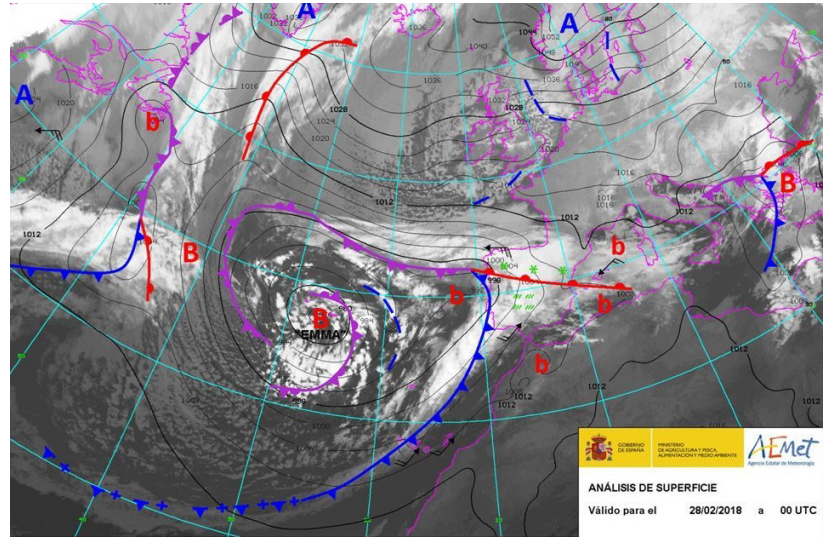

Figura 7. Análisis en superficie sobre imagen del canal infrarrojo del Meteosat 11 del día 28 de febrero a las 00 UTC. muestra un típico perfil de frente cálido (figura 8).
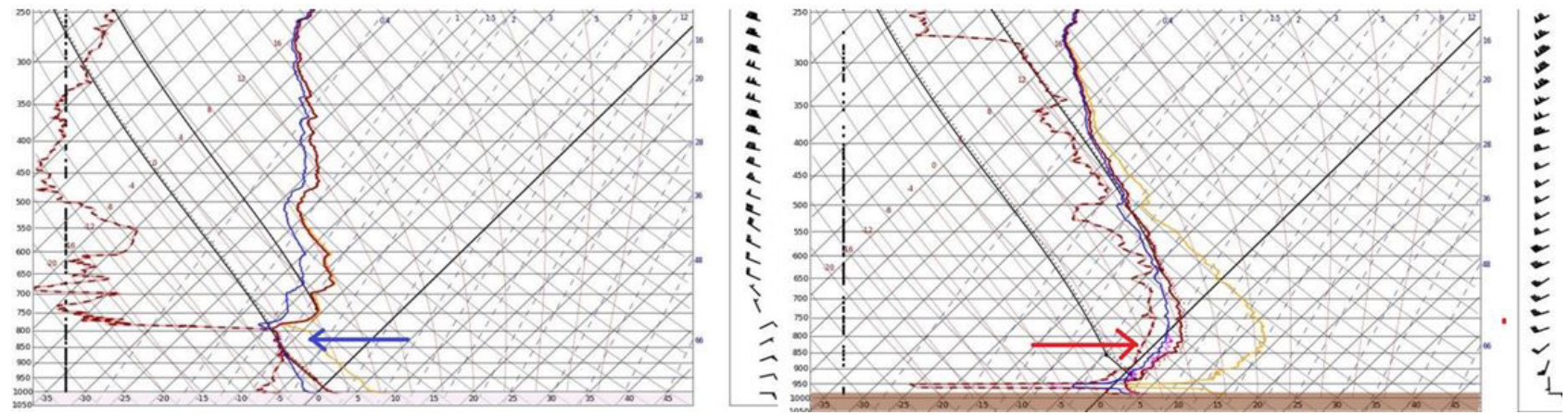

Figura 8. Sondeos de Santander a las 12 UTC del día 27 (izquierda) y del día 28 (derecha). Las flechas indican el descenso (izquierda) y el ascenso térmico (derecha). A la derecha de cada diagrama aparece el viento en la vertical de la atmósfera, donde se ve el cambio de viento asociado al paso del frente cálido.

Con el paso del frente se produjeron las nevadas a nivel del mar en la costa cantábrica y catalana durante las primeras horas del día 28 (figura 9). Debido al rápido ascenso de temperaturas la cota de nieve subió rápidamente de manera que la precipitación pasó a ser en forma de lluvia.

Posteriormente, en su desplazamiento hacia el norte, el frente cálido también produjo importantes nevadas en cotas muy bajas en Francia y en las islas británicas.

Figura 9.

Nieve en Bilbao y en Sant Feliu de

Llobregat, cerca de Barcelona, a primeras horas del día 28 de febrero.
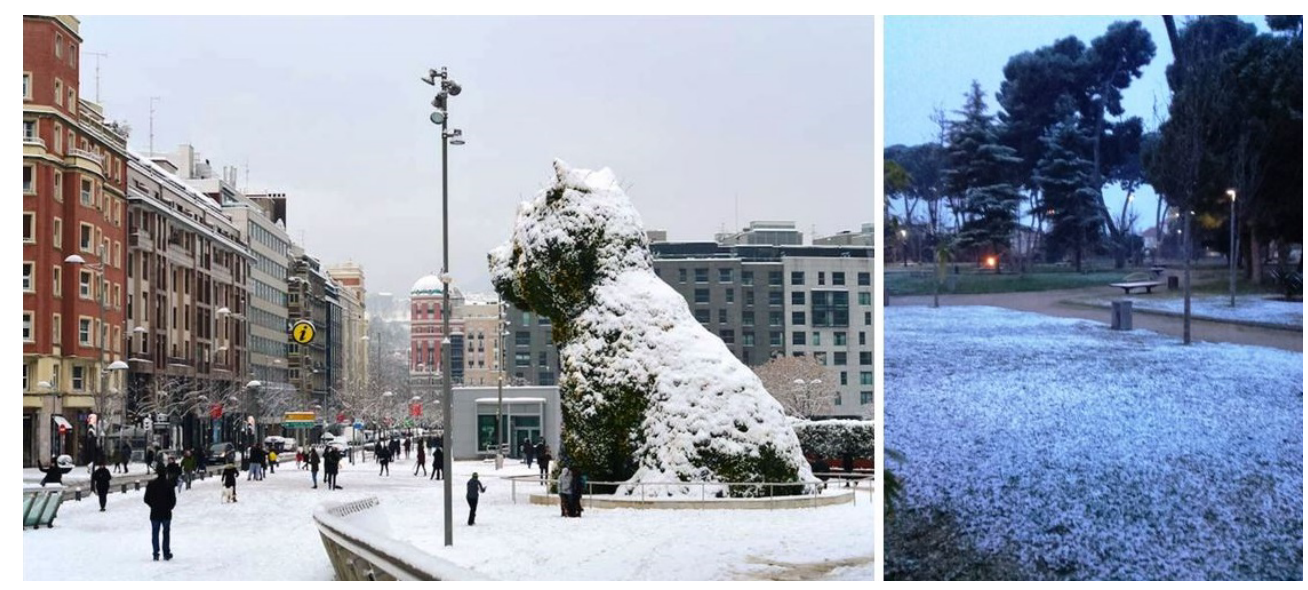


\section{PREDICCIÓN DEL EPISODIO}

Los modelos de predicción utilizados operativamente por AEMET ya anticipaban con bastantes días de antelación tanto las intensas precipitaciones como las nevadas en cotas muy bajas en el norte peninsular. Los dos grandes sistemas que interactuaron, la masa de aire frío y la borrasca Emma, fueron bien previstos tanto en su evolución como en su interacción, tanto espacial como temporalmente.

Como ejemplo se muestra en la figura 10 la previsión de nieve por la pasada de las 00 UTC del día 26 de febrero del modelo determinista IFS del ECMWF desde el mismo día 26 de febrero hasta el 1 de marzo. Aparece la nieve prevista para el mismo día 26 en Cataluña, el inicio de las nevadas de frente cálido en el centro peninsular el día 27 y, con dos días de antelación, el modelo ya predecía nevadas a nivel del mar en la costa cantábrica y catalana para el día 28, con acumulaciones mucho mayores en los macizos montañosos del norte peninsular. Para el día 1, el gran ascenso previsto de la cota de nieve restringía la previsión de nevadas solo a cotas altas del Pirineo central.
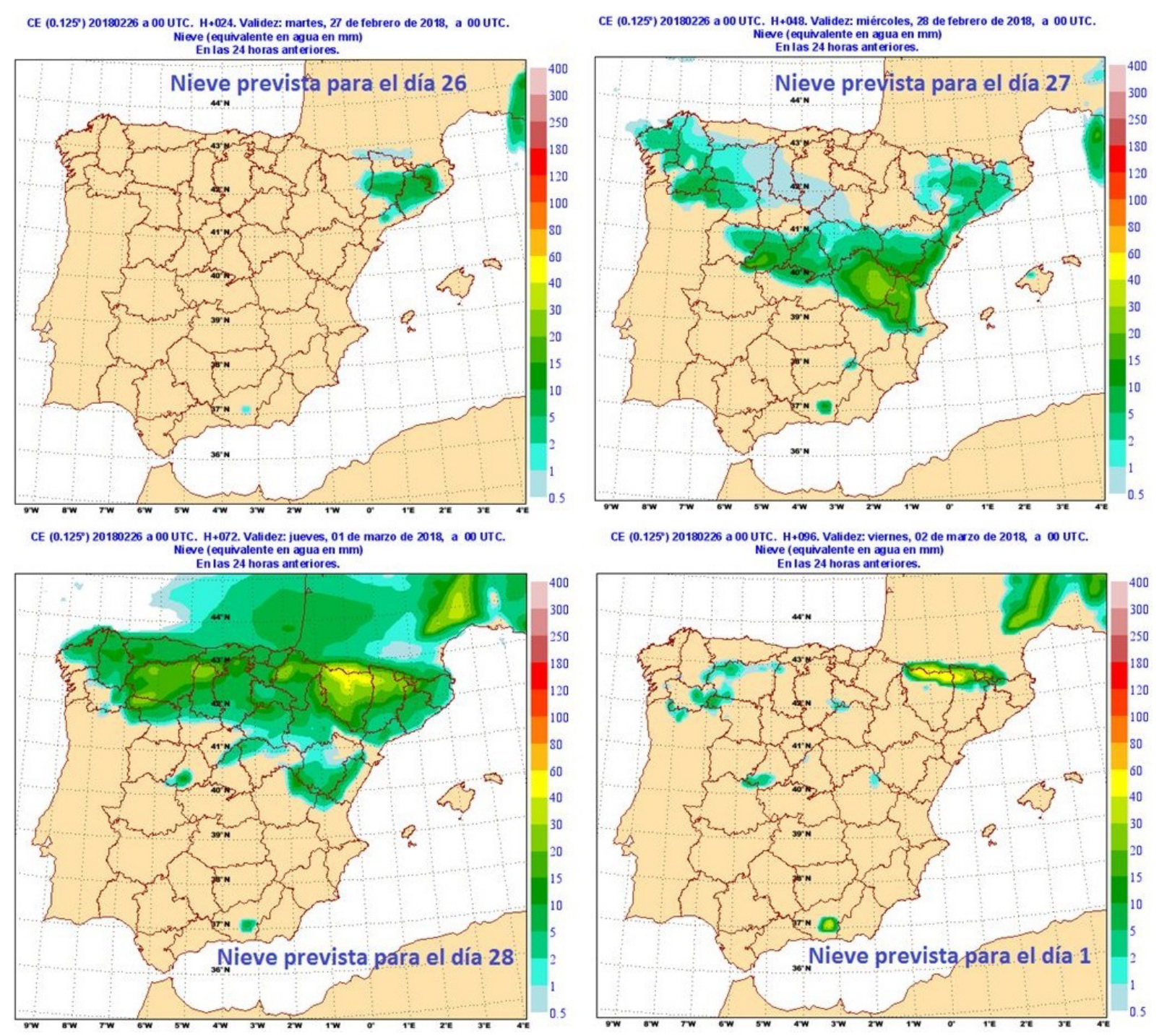

Figura 10. Nieve prevista por la pasada de las 00 UTC del día 26 de febrero del modelo determinista IFS del ECMWF, desde el mismo día 26 de febrero al 1 de marzo.

También el EPS (sistema de predicción por conjuntos) del ECMWF preveía las nevadas a nivel del mar para el día 28 (figura 11). 
EPS $\left.10.25^{\circ}\right) 20180226$ a 00 UTC. H*072. Valider: jueves, 01 de mar 20 de 2018, a 00 UTC.

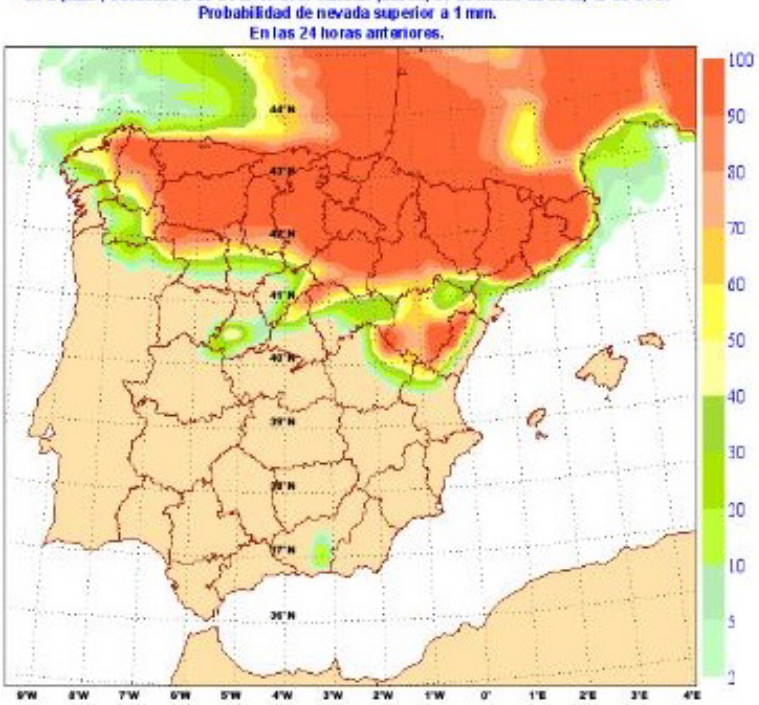

EPS $\left.10.25^{\prime}\right) 20180226$ a 00 UTC. H+072. Valider: jueves, 01 de marze de 2018, a 00 UTC.

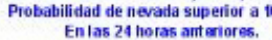

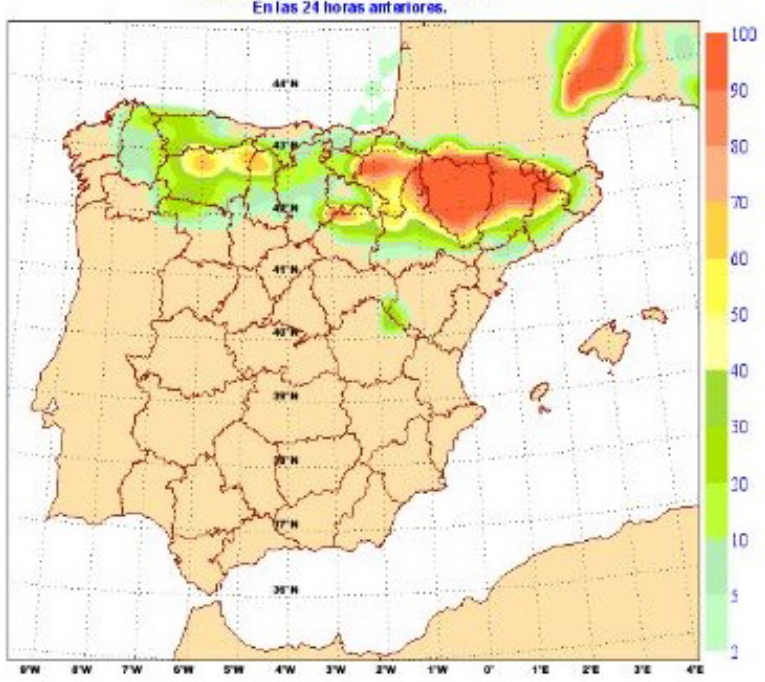

EPS $\left(0.25^{\circ}\right) 20180226$ a 00 UTC. H+072. Vadider: jueves, 01 de mar 20 de 2018 , a 00 UTC.

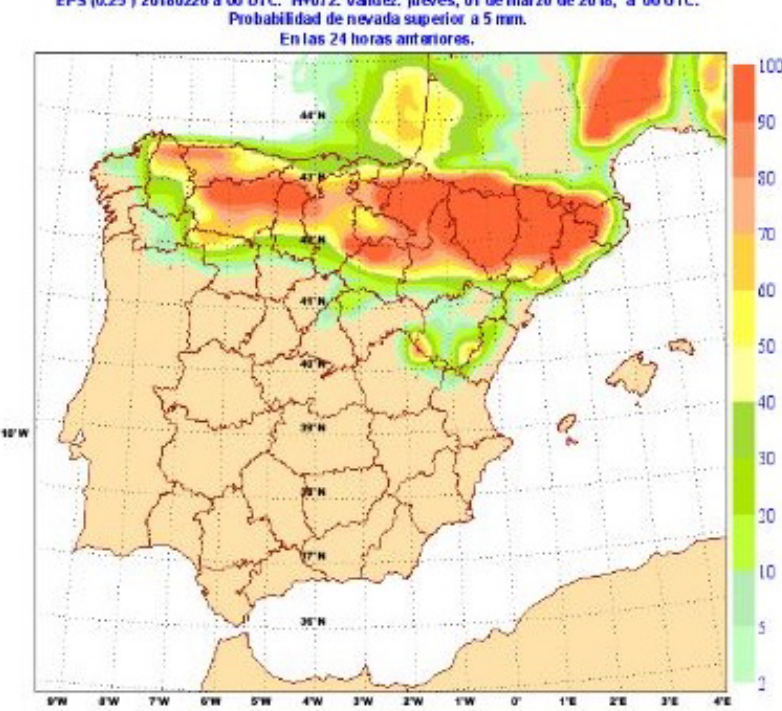

EPS $\left(0.25^{\circ}\right) 20180226$ a 00 UTC. H+072. Valider: jueves, 01 de marzo de 2018, a 00 UTC. Probabilitad de nevada superior a $20 \mathrm{~mm}$.

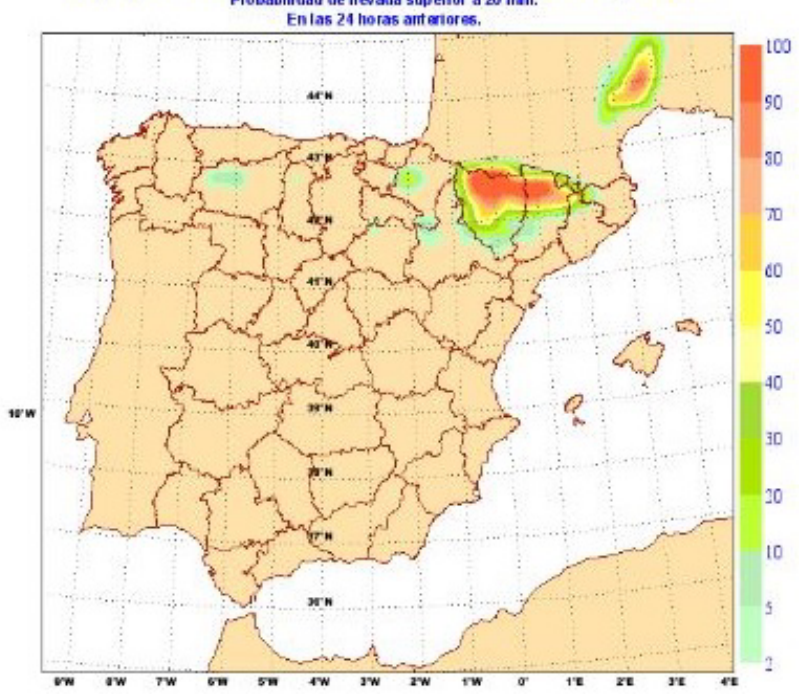

Figura 11. Mapas previstos para el día 28 de probabilidad de nevada superior a $1 \mathrm{~cm}$ (arriba-izquierda), $5 \mathrm{~cm}$ (arriba-derecha), $10 \mathrm{~cm}$ (abajo-izquierda) y $20 \mathrm{~cm}$ (abajo-derecha), por el EPS del ECMWF en su pasada de las 00 UTC del día 26.

Se emitieron con antelación avisos especiales de fenómenos adversos, donde se indicaba que se esperaban nevadas a cualquier cota en el norte de la península ibérica (figura 12).

De acuerdo con las predicciones se emitieron numerosos avisos de nivel amarillo y naranja por acumulaciones de nieve en casi todas las zonas de la mitad norte peninsular, incluyendo las zonas costeras afectadas.

Figura 12. Aviso especial emitido por AEMET el día 26 de febrero.
AGENCIA ESTATAL DE METEOROLOGÍA AVISO ESPECIAL DE FENÓMENOS ADVERSOS AVISO ESPECIAL NÚMERO $14 / 2018$ EMTTIDO A IAS 14:00 HORA OFICIAI DEL 26/2/2018

LA AGENCIA ESTATAL DE METEOROLOGÍA INFORMA: 1.- Fenómeno meteorológico: Lluvia, nieve y viento. 2.- Ámbito geográfico: Península Ibérica y Canarias 3.- Comienzo de la situación: Lunes, día 26.

4.- Duración: al menos una semana.

5.- Grado de probabilidad: Muy probable (mayor del $80 \%$ ).

6.- Descripción de la situación meteorológica: A partir de la tarde de hoy, lunes 26 , la proximidad de diversas borrascas atlánticas a la Península, una de ellas ya nombrada como Emma sobre el Atlántico, dará lugar al inicio de una situación caracterizada por el paso sucesivo de sistemas frontales que, dependiendo de los días, ocasionarán nevadas extensas en cotas muy bajas, lluvias muy abundantes y generalizadas y rachas fuertes o muy fuertes de viento.

Las nevadas estarán asociadas al choque de dos masas de aire de caracteristicas muy diferentes, por un lado la llegada por el nordeste peninsular durante el lunes y martes de una masa de aire polar continental, fría y seca, y por otro la masa de aire atlántico que avanza desde el suroeste, con gran aporte de humedad y mucho más cálida. Durante el martes y miércoles esta situación dará lugar a precipitaciones, inicialmente en forma de nieve a cualquier cota precipitaciones, inicialmente en forma de nieve a cualquier cota en en lluvia de sur a norte a medida que la cota de nieve ascienda con en lluvia de sur a norte a medida que la cota de nieve ascienda con el resto de la semana es muy probable que las nevadas queden restringidas a zonas de montaña. 


\section{LAS NEVADAS EN LA COSTA CANTÁBRICA Y CATALANA. CASO ATÍPICO DE NEVADA DE FRENTE CÁLIDO}

Para averiguar en qué situaciones meteorológicas se suelen producir las nevadas a cotas muy bajas en el litoral catalán y cantábrico, por una parte se ha revisado la bibliografía existente y por otra se han buscado los registros históricos de nevadas destacables en las ciudades de Santander y Barcelona, como representativas del litoral cantábrico y catalán respectivamente. Para los casos de las principales nevadas acontecidas en las últimas décadas se han comparado los mapas de reanálisis, obtenidos del modelo NOAA, con los de la situación en la que se han dado las nevadas de frente cálido de este episodio.

\subsection{Nevadas en la costa cantábrica}

Los estudios anteriores indican que las situaciones de nevadas en la costa cantábrica requieren de la presencia de un anticiclón en el Atlántico norte o Escandinavia, el cual provoca unas advecciones de aire muy frío procedente de altas latitudes. Las masas de aire más propicias son las marítimas árticas, muy frías y relativamente húmedas. Por otra parte las bajas presiones suelen situarse sobre Centroeuropa o en el Mediterráneo occidental (figura 13). En ocasiones las nevadas se producen por el paso de frentes fríos procedentes del norte. El cese de las nevadas se suele producir con la entrada de aire más seco de origen continental.

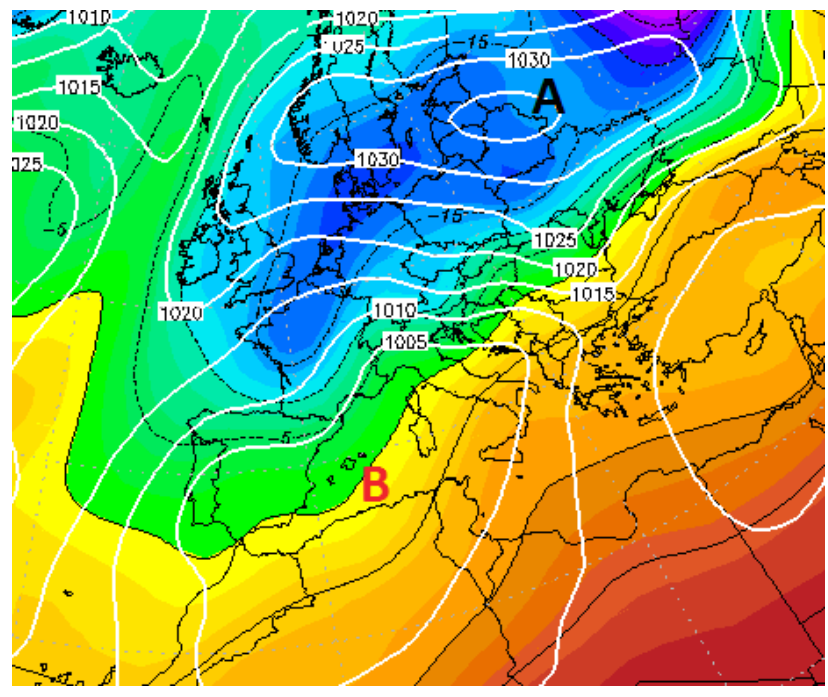

Figura 13.

Reanálisis del mapa isobárico en superficie y de temperatura a $850 \mathrm{hPa}$ del día 27 de noviembre de 1890, con gran nevada en Santander.

Aparece una gran área de altas presiones desde el norte de Rusia hasta las islas británicas y una área de bajas presiones sobre el Mediterráneo occidental.

\subsection{Nevadas en la costa catalana}

En el caso de las nevadas históricas que han afectado la costa catalana también se sitúa un anticiclón en el Atlántico norte o Escandinavia, con la correspondiente advección de aire frío procedente de altas latitudes. Las depresiones también suelen situarse sobre el Mediterráneo occidental o en ocasiones sobre el interior de la península ibérica (figura 14).

Figura 14.

Reanálisis del mapa isobárico en superficie y de temperatura a $850 \mathrm{hPa}$ el día 28 de enero de 1963 en el que se dio una histórica nevada en Barcelona.

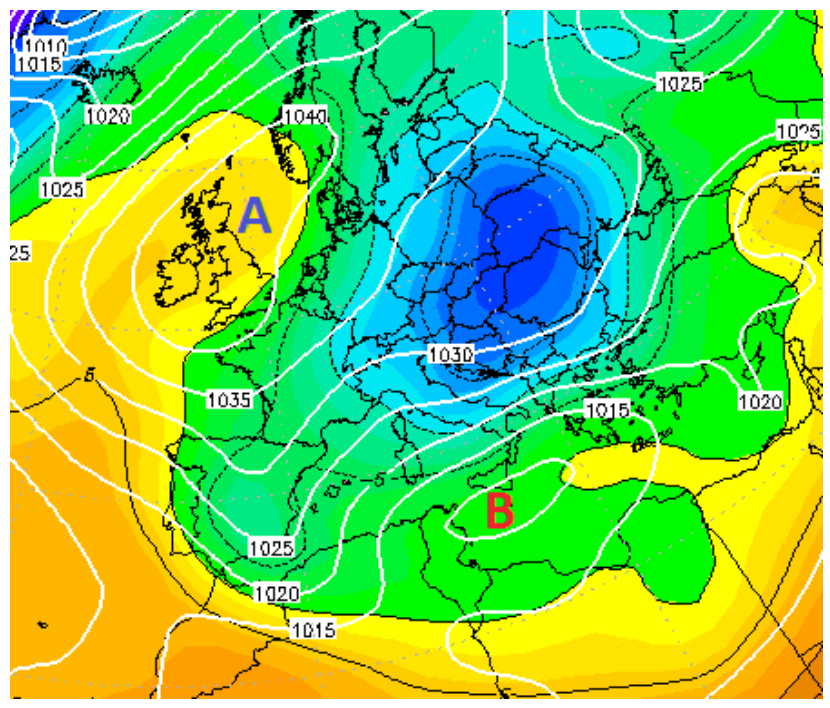

Las nevadas se pueden producir con advecciones de aire frío bastante húmedo por su recorrido marítimo. Pero también se dan casos de nevadas en cotas muy bajas con una masa de aire fría y seca, de manera que son las convergencias en capas bajas asociadas al dipolo orográfico pirenaico las que dan lugar a la formación de nubosidad y precipitaciones. Es el caso de la nevada del día 26 que tuvo lugar al inicio de este episodio (figura 3). 


\subsection{Episodio inusual de nevada de frente cálido}

Por último, en las figuras 15 y 16 se comparan dos situaciones correspondientes a días de importantes nevadas en Santander y en Barcelona, bastante representativas de la situación sinóptica habitual en nevadas en las respectivas zonas costeras, con la situación sinóptica en superficie del día de la nevada del episodio que aquí se expone. El día 28 de febrero de 2018 destaca la presencia de una baja al oeste peninsular y el frente cálido que la atraviesa de sur a norte.
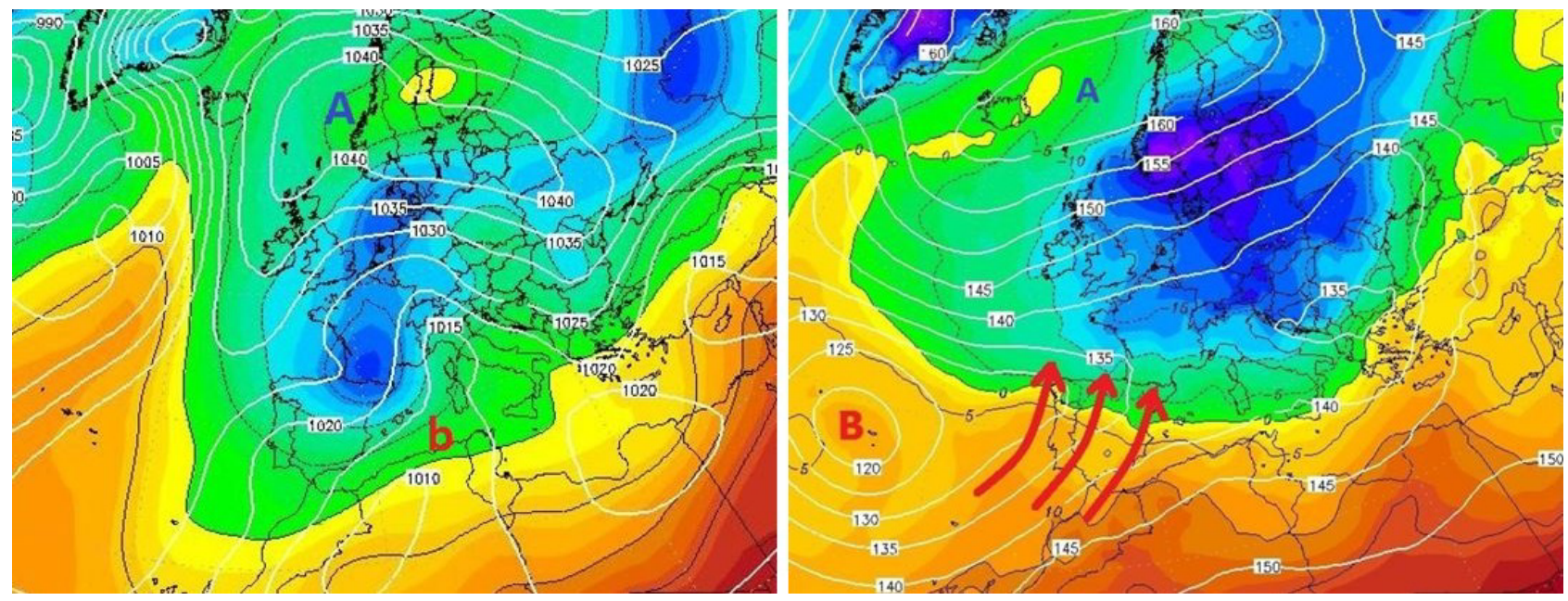

Figura 15. Comparación entre los mapas de presión en superficie correspondientes a los días

15 de enero de 1985 (izquierda) y 28 de febrero de 2018 (derecha), ambos con nevadas en Santander.
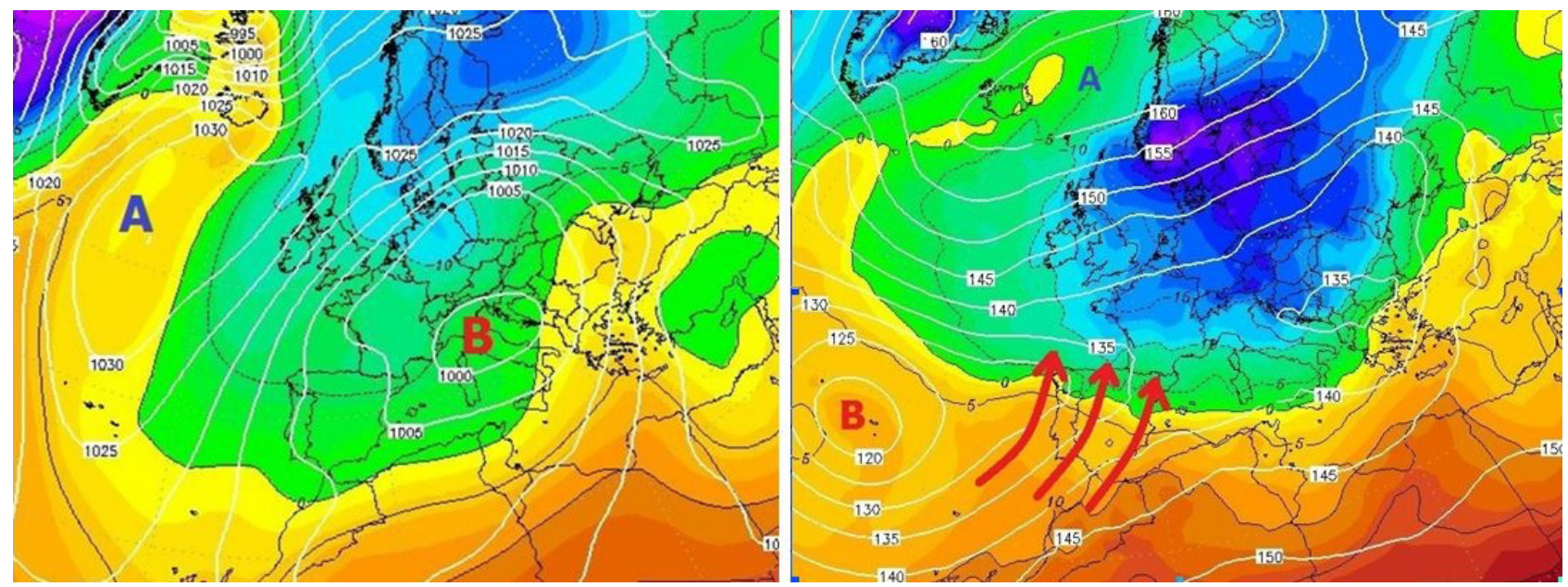

Figura 16. Comparación entre los mapas de presión en superficie correspondientes a los días 8 de febrero de 1983 (izquierda) y 28 de febrero de 2018 (derecha), ambos con nevadas en Barcelona.

\section{CONCLUSIONES}

El día 28 de febrero de 2018 tuvo lugar un episodio de nevadas en la costa cantábrica y catalana provocado por el paso de un frente cálido que se formó por la interacción de una masa de aire de origen polar continental y la borrasca denominada Emma. Este frente se formó sobre la península ibérica y frenó el avance de la masa de aire frío hacia el sur. En su desplazamiento hacia el norte, el frente cálido dio lugar a nevadas en cotas muy bajas del tercio norte peninsular, donde aún se encontraba el aire muy frío en capas bajas de la atmósfera. 
Las nevadas al nivel del mar en Cataluña y en la costa cantábrica son poco frecuentes y se suelen producir con depresiones situadas en el entorno del Mediterráneo occidental y con advecciones frías más o menos húmedas procedentes del norte. Por ello se puede considerar como excepcional este episodio de nevadas motivadas por el paso de un frente cálido procedente del sur y con una borrasca situada sobre el Atlántico al oeste de la península ibérica.

\section{REFERENCIAS}

Agencia Estatal de Meteorología (AEMET), 2018. Avance climatológico mensual de febrero de 2018. Delegación Territorial de AEMET en Cataluña.

Fernández Guardia, A., 2016. Meteorological characterization of exceptional snowfall events. Treball fi de grau. Facultat de Física, Universitat de Barcelona.

MÉTÉo FrANCE. Bilan climatique de l'hiver 2017-2018.

Ortiz Berenguer, F. J., 1998. Predicción y evaluación de la cota de nieve en la cordillera cantábrica. Nota Técnica n. ${ }^{\circ}$ 8. Sección de Estudios y Desarrollos. CMT en Cantabria y Asturias, INM.

Puente, J. M., 2009. La nieve en la ciudad de Santander (1880-2006). Publicado en tiempo.com.

Puente, J. M., 2006. La nieve en la ciudad de Bilbao (1880-2006). Publicado en tiempo.com.

Servei Meteorològic de Catalunya, 2018. Butlletí climàtic mensual. Febrer del 2018.

Los mapas de reanálisis del NOAA han sido obtenidos de wetterzentrale.de. 